\title{
Laboratory-Scale Photomineralization of $n$-Alkanes in Gaseous Phase by Photocatalytic Membranes Immobilizing Titanium Dioxide
}

\author{
Ignazio Renato Bellobono, ${ }^{1}$ Rodica Stanescu, ${ }^{2}$ Cristina Costache, ${ }^{2}$ Carmen Canevali, ${ }^{3}$ Franca Morazzoni, ${ }^{3}$ \\ Roberto Scotti, ${ }^{3}$ Riccardo Bianchi, ${ }^{4}$ Emilia Simona Mangone, ${ }^{5}$ Giulia de Martini, ${ }^{5}$ and Paola Maria Tozzi ${ }^{5}$ \\ ${ }^{1}$ Environmental Research Centre, University of Milan, Via C. Golgi 19, 20133 Milan, Italy \\ ${ }^{2}$ Department of Inorganic Technology and Environmental Protection, Faculty of Industrial Chemistry, \\ Polytechnic University of Bucharest, 011061 Bucharest, Romania \\ ${ }^{3}$ Department of Materials Science, University of Milano Bicocca, 20126 Milan, Italy \\ ${ }^{4}$ CNR, ISTM, Institute of Molecular Sciences and Technologies, 20133 Milan, Italy \\ ${ }^{5}$ R\&D Group, BIT srl, 20121 Milan, Italy
}

Received 19 February 2006; Accepted 4 May 2006

\begin{abstract}
Kinetics of photocatalytic oxidation of methane, ethane, and $n$-heptane, to yield intermediates, and photomineralization of intermediates, to yield carbon dioxide and water, was studied in the gaseous phase, at $308 \pm 2 \mathrm{~K}$, by a laboratory-scale photoreactor and photocatalytic membranes immobilizing $30 \pm 3 \mathrm{wt} . \%$ of $\mathrm{TiO}_{2}$, in the presence of aerosolized stoichiometric hydrogen peroxide as oxygen donor, and at a relative humidity close to $100 \%$. The whole volume of irradiated solution was $4.000 \pm 0.005 \mathrm{~L}$, the ratio between this volume and the geometrical apparent surface of the irradiated side of the photocatalytic membrane was $3.8 \pm 0.1 \mathrm{~cm}$, and the absorbed power was $0.30 \mathrm{~W} / \mathrm{cm}$ (cylindrical geometry). The pinetic parameters of the present work substantially coincide with those of the same molecules previously studied in aqueous solution, within the limits of experimental uncertainty. Photocatalytic processes thus appear to be controlled by interface phenomena, which are ruled kinetically, and apparently also thermodynamically, by concentration gradients, independently on diffusion and other processes in the aqueous or gaseous bulk, if turbulence in these phases is adequately assured.
\end{abstract}

Copyright () 2006 Ignazio Renato Bellobono et al. This is an open access article distributed under the Creative Commons Attribution License, which permits unrestricted use, distribution, and reproduction in any medium, provided the original work is properly cited.

\section{INTRODUCTION}

The presence of harmful pollutants in gaseous emissions is notoriously a topic of global concern. Conventional methods of treatment, such as stripping, result only in transfer from one phase to another, and not to complete destruction of pollutants. Other processes, such as wet air oxidation, incineration, and so on, require high concentrations of pollutants, and/or addition of fuels. Heterogeneous photocatalysis, on the contrary, is a very promising method for transformation of toxic organic compounds, present in gaseous streams, particularly, into harmless final species, such as carbon dioxide and water $[1,2]$, and can be carried out also by exploiting solar energy. Probably, the major problem in photocatalysis, from the point of view of industrial applicability, is given by the necessity of immobilizing the photocatalyst, by firmly anchoring it to a suitably reacting structure, and of reaching the highest performance as possible in photocatalytic reactors, in continuous mode [3]. These two aspects (kind of immobilisation and quantum yields) need therefore to be considered as priority options for engineering applications. In all preceding papers of this series (see, e.g., among the most recent ones $[4,5])$ the main concern has been devoted to these topics, and immobilisation of photocatalyst and its photopromoting agents in a membrane structure has been the choice in order to realize modular membrane reactors, the performance of which has been effectively estimated as one of the highest for energy efficiency [3].

In preceding works of this series, the $\mathrm{TiO}_{2}$-mediated photomineralization of $n$-alkanoic acids $[6,7]$ and of $n$-alkanols 
[8] was investigated at a laboratory-scale level, in aqueous solutions. As quantum yields of photomineralization have been found independent of radiation wavelength, within the absorption range of semiconductor, but dependent on radiant power, the best way to compare different sets of experiments is to operate with a radiant power as low as possible, in the range $0.1-0.4 \mathrm{~W} / \mathrm{cm}$, in which limiting quantum yields are maximised and become independent of radiant power. For this reason, in a successive work [9], experiments on $n$-alkanes, in aqueous solutions, were carried out in these conditions, with polychromatic irradiation at $0.30 \mathrm{~W} / \mathrm{cm}$ of absorbed power per unit length of photocatalytic membranes.

To interpret experimental data, a kinetic model was set up and employed, by which mineralisation of substrate to $\mathrm{CO}_{2}$ was supposed to occur, by kinetic constants $k_{1}$, through one single intermediate, mediating the behaviour of all the numerous real intermediates formed in the path from the substrate to $\mathrm{CO}_{2}$ (kinetic constants of formation of the latter being $k_{2}$ ). A competitive Langmuirian adsorption of both substrate and "intermediate" was also supposed to be operative, as expressed by apparent adsorption constants $K_{1}$ and $K_{2}$, possessing a, partly at least, kinetic significance. By Langmuir-Hinshelwood treatment of initial rate data, starting values of the $k$ and $K$ couples were obtained, from which, by a set of differential equations, the final optimised parameters, $k_{1}$ and $K_{1}, k_{2}$ and $K_{2}$, were calculated, able to fit the whole photomineralization curve, and not only its initial segment, as the Langmuirian parameters do.

In the present paper, the same kinds of experiments were carried out by using methane, ethane, and $n$-heptane as substrates, but by operating entirely in the gas phase. A 100\% relative humidity was maintained, in the presence of aerosolized stoichiometric hydrogen peroxide as oxygen donor, and the experimental concentration range of these $n$-alkanes was extended to higher values than those tested in the aqueous solutions investigated in a previous paper [9]. The same kinetic model with four parameters was utilized, in order to check its fitness also during the gas phase photomineralization.

\section{EXPERIMENTAL DETAILS}

\subsection{Materials}

Methane, ethane, and $n$-heptane were obtained from Fluka (GC purity greater than $99.8 \%$ ). They were used as received with no further purification. Solutions of hydrogen peroxide, to be aerosolized in the reactor, as stoichiometric oxygen donor, and to maintain a $100 \%$ relative humidity at the operating temperature of the photoreactor, were prepared with ultra pure water (maximum contents of $\mathrm{Na}^{+}$and heavy metal ions 0.02 and $0.004 \mathrm{mg} \mathrm{Kg}^{-1}$, resp.): this was obtained by cross-flow ultrafiltration on composite membranes immobilizing active carbon and nuclear grade ion exchange resins, as described in [10]. Concentration of substrates in the gas phase was 10.0-1000 ppm (mass expressed as carbon/volume) for all of the alkanes studied.

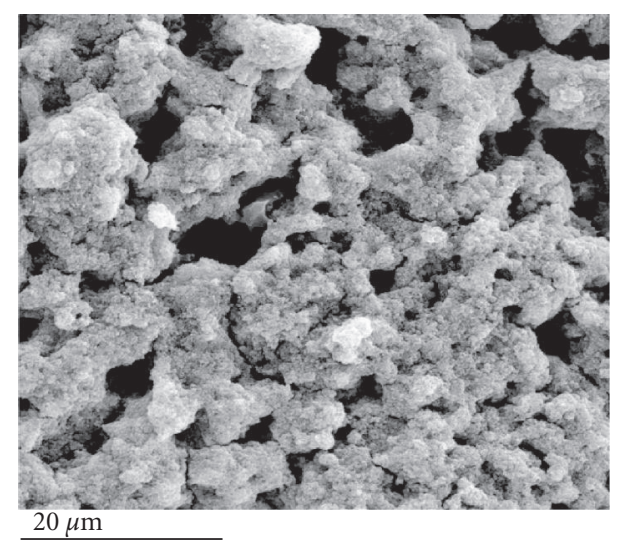

Figure 1: Electron micrograph (scale shown on figure) of the surface top layer of a membrane (PHOTOPERM ${ }^{\circledR}$ BIT/313) prepared by photoinitiated grafting and graft-polymerization, onto a nonwoven polypropylene tissue of $250 \mathrm{~g} \cdot \mathrm{m}^{-2}$ of a prepolymeric blend containing $30 \pm 3 \mathrm{wt}$. of titanium dioxide (P25 by Degussa, Germany), in the presence of proprietary photoinitiating and photosensitizing systems.

\subsection{Photocatalytic membranes}

The photocatalytic membranes (PHOTOPERM ${ }^{\circledR}$ BIT/313), kindly supplied by BIT srl (Milan, Italy) were standard photocatalytic membranes, immobilizing $30 \pm 3 \mathrm{wt} . \%$ of titanium dioxide (P25 by Degussa, Germany) and no photocatalytic promoter, as cocatalyst. Photocatalytic membranes [11, 12], and more generally membranes, prepared by photografting, to immobilise reagents, catalysts, and sorbents [13-15], were described and characterised in previous studies. These membranes were prepared (patents pending) by grafting and graft-polymerising, onto a nonwoven polypropylene tissue, $250 \pm 9 \mathrm{~g} \cdot \mathrm{m}^{-2}$ of a prepolymeric blend containing $30 \pm 3 \mathrm{wt} . \%$ of titanium dioxide (P25 by Degussa, Germany), under rheological control, in the presence of proprietary photoinitiating and photosensitizing systems. Electron micrograph of the surface top layer of one of these membranes is shown in Figure 1. Aggregates of the titanium dioxide nanopowder, anchored and immobilised onto the microporous channels of the membrane may be clearly observed.

\subsection{Apparatus and procedures}

The laboratory-scale photoreactor was the same as that already described [11], with the only difference that it was made gas tight to avoid any leakage of substrate and/or intermediates through the vapour phase. Furthermore, $150 \mathrm{~mL}$ of an aqueous solution of hydrogen peroxide were aerosolised continuously into the reactor, in order to supply the stoichiometric amount of this oxygen donor, as well as to maintain a $100 \%$ relative humidity at $308 \pm 2 \mathrm{~K}$, which was the operating temperature of the photoreactor. Overall volume of gaseous phase being treated was $4.000 \pm 0.005 \mathrm{~L}$; the ratio between the overall reacting volume and the apparent, 
geometrical surface area of the irradiated side of the membrane was $3.8 \pm 0.1 \mathrm{~cm}$. A high pressure mercury arc lamp with a nominal power of $0.12 \mathrm{KW}$ was employed, kindly supplied by Chimia Prodotti e Processi (Muggiò, Milan, Italy): it was further provided with a step by step power regulation device, so that power absorbed by the membrane, as determined actinometrically, could be varied between 0.10 and $1.05 \mathrm{~W} \cdot \mathrm{cm}^{-1}$. In all the experiments, it was fixed at $0.30 \mathrm{~W} \cdot \mathrm{cm}^{-1}$. The membrane being concentric to the lamp in the photoreactor used, absorbed power per unit length of lamp was also the same, if referred to unit length of membrane. At $0.30 \mathrm{~W} \cdot \mathrm{cm}^{-1}$ the overall power absorbed by the membrane, within the absorption range of immobilized semiconductor, in the experimental conditions of the present work, was $7.50 \mathrm{~W} \cdot \mathrm{cm}^{-1}$, corresponding to $1.10 \times 10^{-5}$ Einstein $s^{-1}$. In this paper, as well as in all preceding papers of this series, when concerning laboratory-scale experiments carried out in the presence of hydrogen peroxide as oxygen donor, the radiating flux, unless otherwise stated, being filtered by a suitable borosilicate glass, corresponds to a radiation wavelength range from the band gap of $\mathrm{TiO}_{2}$ downwards to $315 \mathrm{~nm}$, so that direct photolysis of hydrogen peroxide, to give hydroxyl radicals, is inhibited. Owing to the refrigeration system of the lamp, by means of water circulating in the lamp sheath, made of quartz, mean temperature, during the runs, was $308 \pm 2 \mathrm{~K}$.

In the present, as well as in previous papers of this series, membranes were placed and fixed coaxially with the lamp, in such a way that the flow was perpendicular to the membrane. Owing to the microporous structure of the membrane (see Section 2.2), the pressure drop was very modest: it corresponded to $0.9-1.6 \mathrm{~mm} \mathrm{Hg}$ when the flow rate of the gaseous system was $4 \mathrm{~m}^{3} / \mathrm{h}$ in the laboratory-scale photoreactor.

The initial rate $r_{0}$ of photodegradation of alkanes was evaluated from curves of their concentration versus time in the linear range, where zero-order kinetics was apparent. Experiments were repeated for each set of conditions, essentially for each value of initial concentration $C_{0}$ of micropollutant, so that the mean initial rate and its standard deviation could be estimated.

The disappearance of the alkanes, as such, was followed by GC-MS quantitative analysis into the injection port of a GC-MS system. A Varian 3400 with a septum equipped programmable injector was used. In the GC oven, a $30 \mathrm{~m} \times$ $0.53 \mathrm{~nm}$ DB5MS megabore column was installed with the following temperature programme: $20^{\circ} \mathrm{C}$ for 1 minute followed by a temperature raise of $5^{\circ} \mathrm{C} / \mathrm{min}$ to $240^{\circ} \mathrm{C}$, which was held for 4 minutes.

As carrier gas He was used at a flow rate of $25 \mathrm{~mL} / \mathrm{min}$. The GC was interfaced via jet-separator ( 0.3 torr in the separator and 0.03 torr in the analyser) and a transfer-line $\left(270^{\circ} \mathrm{C}\right)$ to an ITS40 ion trap mass-spectrometer (ITMS). The ion trap was operated at $300^{\circ} \mathrm{C}$ in the electron impact mode, scanning from $35 \mathrm{M} / \mathrm{Z}$ to $550 \mathrm{M} / \mathrm{Z}$ in 1.5 second. The GC-ITMS data were acquired on a 386/387 Compaq personal computer with Saturn I software (Varian). The alkane was identified by its mass spectrum and standard samples. Quantification was based on seven-point calibration curves using, as internal standard, a standard sample containing the perdeuterated alkanes.

In order to study the relationship between rate of alkane degradation, as such, and the overall rate of organic carbon disappearance, this latter was also followed, by total organic carbon (TOC) analysis. To this purpose, a Shimadzu TOC-V instrument was used, by which the contribution of inorganic carbon present was always evaluated in order to obtain TOC by difference from total carbon and inorganic carbon determinations. Determinations were accurate within $30 \mathrm{ppb}$ C.

During all the duration of the runs, no decrease of activity of the immobilised photocatalyst was observed, as checked both by random repetition of experiments at various initial concentrations of the hydrocarbons in the gaseous phase, and by reiteration of whole series of tests at the beginning and at the end of the prolonged period, throughout which experimentation has been carried out.

\subsection{Kinetic modeling}

Kinetic modeling was carried out as described in a preceding paper [4].

\section{RESULTS AND DISCUSSION}

Photomineralization of methane, ethane, and $n$-heptane in gaseous phase at $100 \%$ relative humidity, and in a concentration range corresponding to $10.0-1000 \mathrm{ppm}$ (mass/volume) of carbon, for all the examined substrates, was studied at $308 \pm 2 \mathrm{~K}$, in a laboratory-scale reactor, by polychromatic irradiation at $0.30 \mathrm{~W} / \mathrm{cm}$ (see Section 2), in the presence of aerosolized stoichiometric hydrogen peroxide as oxygen donor, by photocatalytic membranes immobilizing $30 \pm 3 \%$ of titanium dioxide. Kinetics of both substrate disappearance, to yield intermediates, and total organic carbon (TOC) disappearance, to yield carbon dioxide, were followed.

By employing a kinetic model thoroughly described in preceding papers [4], mineralisation of substrate $\mathbf{S}$ to $\mathrm{CO}_{2}$ was supposed to occur through one single intermediate I, mediating the behaviour of all possible intermediates formed in the way from $\mathbf{S}$ to $\mathrm{CO}_{2}$, following Scheme (1):

$$
\mathbf{S} \longrightarrow \mathrm{I} \longrightarrow \mathrm{CO}_{2}
$$

and to imply that both $\mathbf{S}$ and I showed a competitive apparent Langmuirian adsorption onto the immobilized semiconductor, as expressed by apparent adsorption constants $K_{1}$ and $K_{2}$, respectively, while the kinetic constants $k_{1}$ and $k_{2}$ articulate the degradation of $\mathbf{S}$ and $\mathbf{I}$, respectively. When both $k$ and $K$ couples, for substrate and "intermediate," are accessible experimentally, as in the present work, that is when initial rate data, relative to substrate, and TOC as well have been measured, they may be first treated by the classical LangmuirHinshelwood equation. The respective values drawn by the Langmuirian model are unable to describe the whole curve of mineralisation. They may be used, however, as starting values of the model represented by Scheme (1) to draw from the differential equations of this model an optimisation of the whole temporal kinetic curves of substrate and TOC, until 


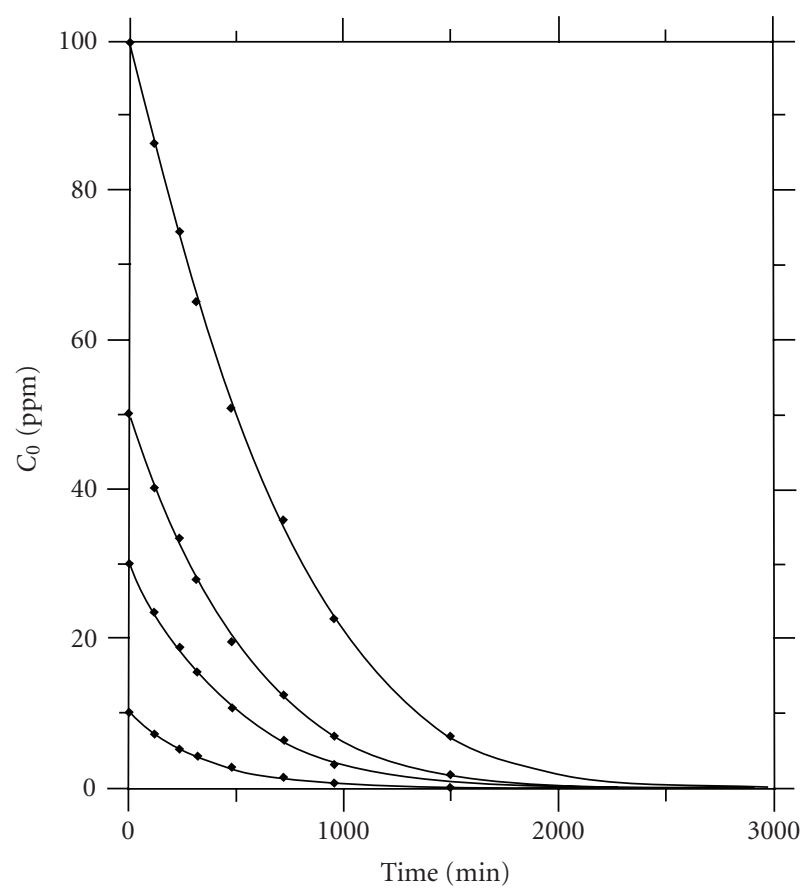

Figure 2: Kinetics of disappearance of methane, as substrate, from the gas phase. Concentrations, $C_{0}$ in $\mathrm{ppm}$, of methane are expressed as carbon, which was not transformed into intermediates, as a function of time, expressed in min, as time necessary to treat $1 \mathrm{~L}$ of gas phase.

consistency is reached and the best fitting of experimental curves is obtained.

Treating of experimental data by the model above [4] allows, consequently, to obtain two couples of parameters, $k_{1}$ and $K_{1}, k_{2}$ and $K_{2}$, which fit the whole photomineralization curve, and not only its initial segment as the Langmuirian parameters generally do. The very satisfactory fitting of experimental kinetic data by this model, notwithstanding its extreme simplicity, is shown in Figures 2 and 3 for the kinetic curves of disappearance of methane as such, and of its TOC disappearance, respectively, in Figures 4 and 5 for the kinetic curves of disappearance of ethane as such, and of its TOC disappearance, respectively, as well as in Figures 6 and 7 for the kinetic curves of disappearance of $n$-heptane as such, and of its TOC disappearance, respectively, at the various initial concentrations specified for the three substrates.

The optimized values for the two $k$ and $K$ couples above, relative to all the three alkanes examined in the present paper in gaseous phase, are reported in Table 1, together with the corresponding data of the same $n$-alkanes as measured in aqueous solution [9] taken from previous work for comparison.

From the observation of these values, some interesting considerations may be drawn as follows.

(a) First of all, the four parameters above obtained from data of the present paper, relative to photodegradation and photomineralization of $n$-alkanes in the gas phase,

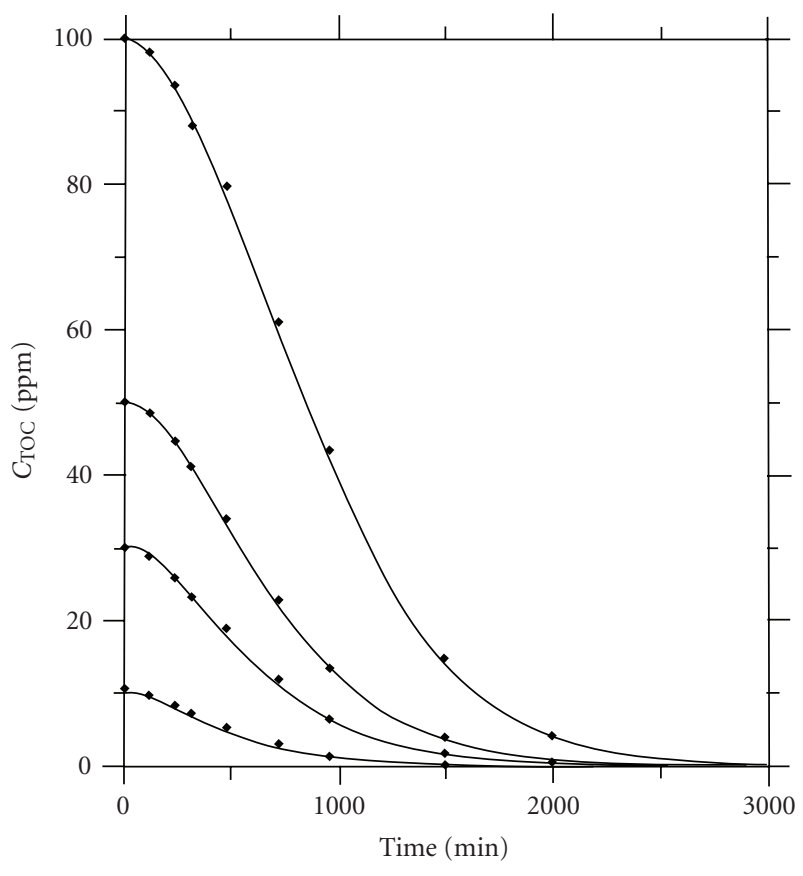

FIGURE 3: Kinetics of disappearance of total organic carbon (TOC) from gaseous methane in the photoreactor, expressed as concentrations, $\mathrm{C}_{\mathrm{TOC}}$ in ppm of carbon, which was not mineralized, as a function of time, expressed in min, as time necessary to treat $1 \mathrm{~L}$ of gas phase.

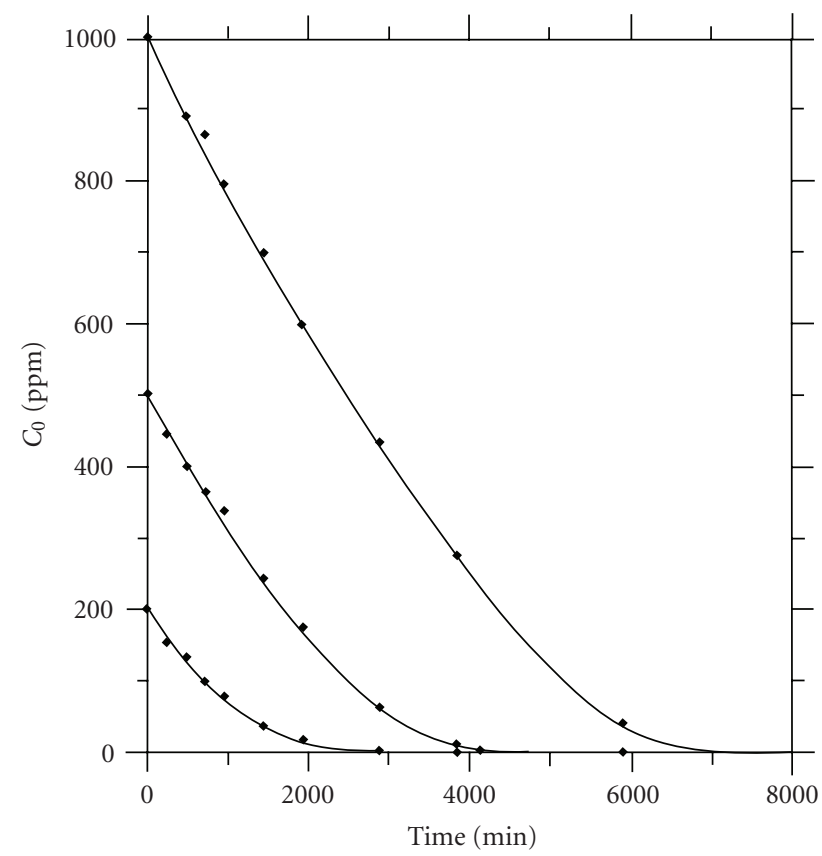

Figure 4: Kinetics of disappearance of ethane, as substrate, from the gas phase. Concentrations, $C_{0}$ in ppm, of ethane are expressed as carbon, which was not transformed into intermediates, as a function of time, expressed in min, as time necessary to treat $1 \mathrm{~L}$ of gas phase. 


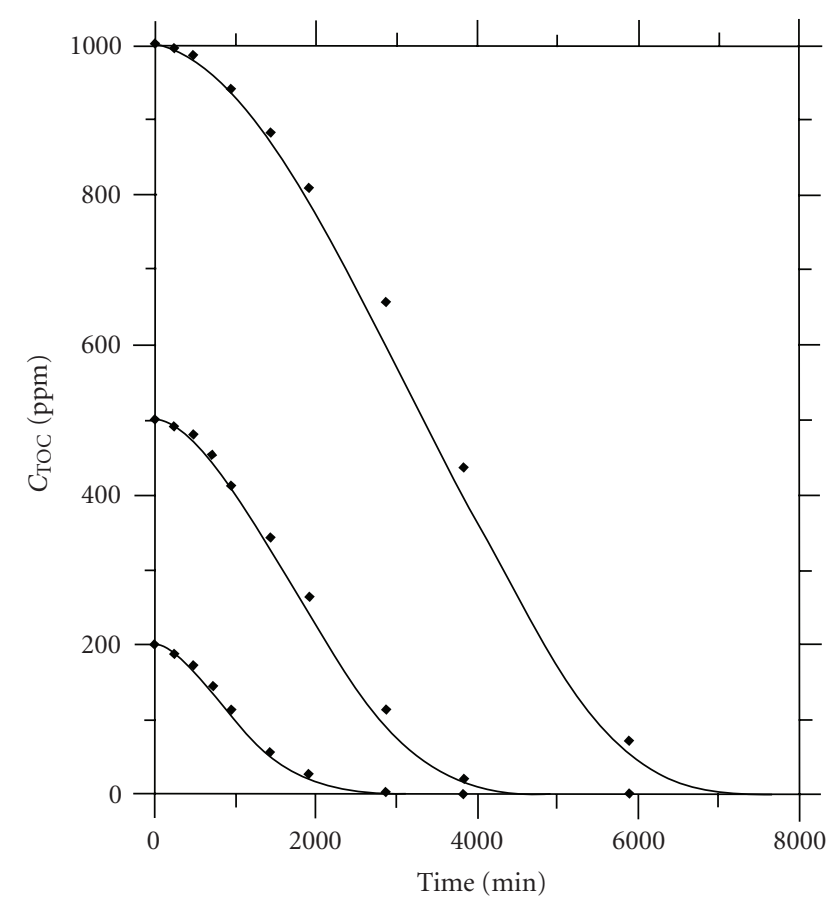

Figure 5: Kinetics of disappearance of total organic carbon (TOC) from gaseous ethane in the photoreactor, expressed as concentrations, $\mathrm{C}_{\mathrm{TOC}}$ in ppm of carbon, which was not mineralized, as a function of time, expressed in min, as time necessary to treat $1 \mathrm{~L}$ of gas phase.

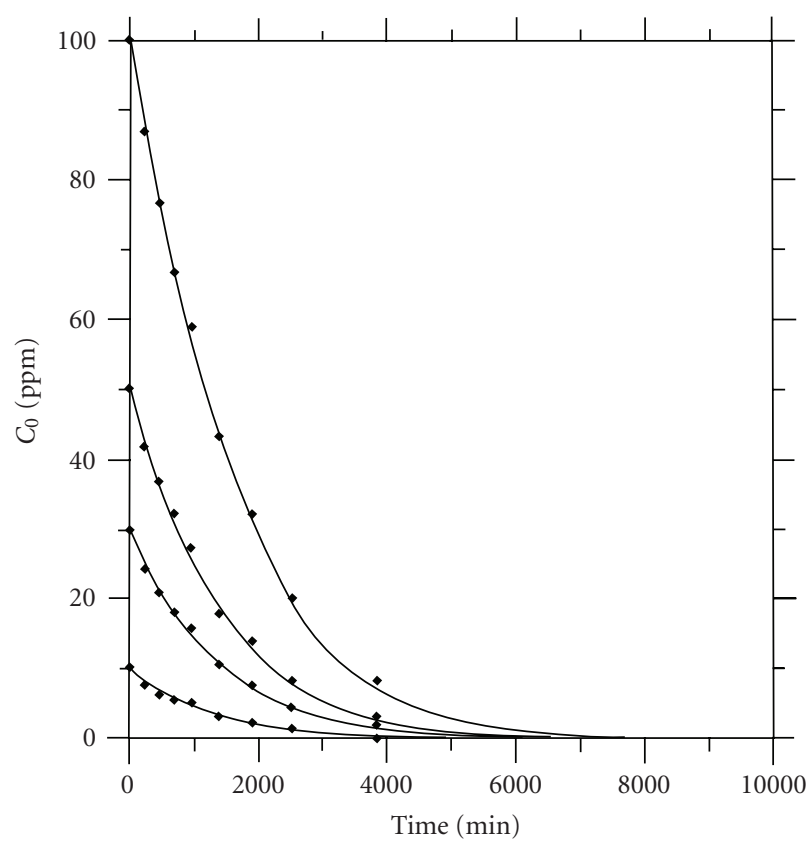

FIgURE 6: Kinetics of disappearance of heptane, as substrate, from the gas phase. Concentrations, $C_{0}$ in ppm, of heptane are expressed as carbon, which was not transformed into intermediates, as a function of time, expressed in min, as time necessary to treat $1 \mathrm{~L}$ of gas phase.

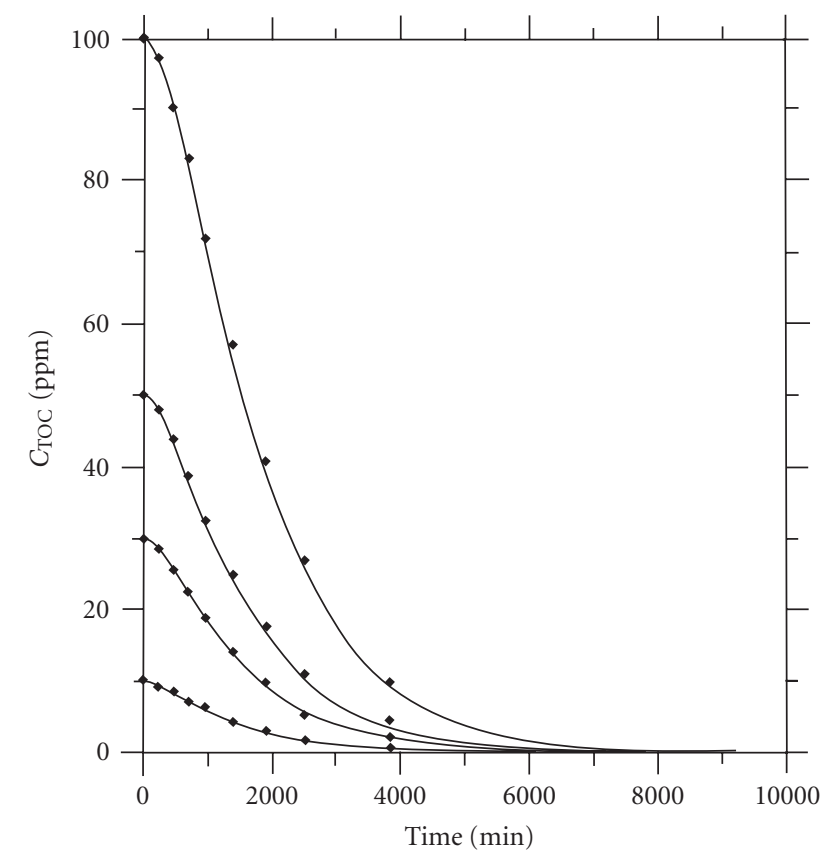

FIGURE 7: Kinetics of disappearance of total organic carbon (TOC) from gaseous heptane in the photoreactor, expressed as concentrations, $\mathrm{C}_{\mathrm{TOC}}$ in ppm of carbon, which was not mineralized, as a function of time, expressed in min, as time necessary to treat $1 \mathrm{~L}$ of gas phase.

substantially coincide within the limits of experimental uncertainty with those measured during experiments carried out in aqueous solution [9], both kind of experiments being performed in conditions of turbulent flow. In these conditions, which also correspond to situations in which the maximum allowable quantum yields or efficiency values very near to these yields are obtained [9], no sizeable or significant difference is thus shown between liquid- and gas-phase photocatalytic experiments concerning the same molecules. At least, this behaviour is surely shown by $\mathrm{TiO}_{2} \mathrm{immobilised} \mathrm{in}$ photocatalytic membranes.

(b) By operating in a gaseous phase saturated by water vapour, as has been done in the present paper, mineralisation proceeds smoothly and completely, as it occurs in the liquid phase [9], and as may be observed in Figures 3, 5 , and 7. On the contrary, if relative humidity is much less than $100 \%$, and particularly when using chlorinated hydrocarbons as substrates [16], the formation of some amounts of relatively photostable intermediates, such as carbon monoxide and phosgene, has been detected. Complete mineralisation was thus sensibly retarded. This inconvenience, however, may be completely obviated, other than maintaining a high humidity in the reaction medium, also by adding suitable photopromoting agents to the membrane [16].

(c) By following the same line of thoughts, as that deriving from (a) above, we may guess that, onto photocatalytic membranes, reactions at the interface control the process. In 
TABle 1: Parameters, $k_{1}$ and $K_{1}, k_{2}$ and $K_{2}$ (uncertainties are indicated between parentheses and expressed, relatively to the last digits, as probable errors of the means of optimized values from nine sets of runs in the range of concentrations tested), are able to fit the whole photomineralization curve, following Scheme (1). They were obtained by optimisation of kinetic curves, by using the set of differential equations corresponding to kinetic model of Scheme (1) [4]. All the parameters are expressed in ppm of carbon units (mass of C/volume) and refer to the photodegradation of $n$-alkanes in gaseous phase (present work), and in aqueous solutions [9], in the presence of stoichiometric hydrogen peroxide as oxygen donor (treated volume of the gas phase or of the aqueous solutions $4.00 \mathrm{~L}$; radiant power of polychromatic high pressure mercury arc lamp absorbed by the photocatalytic membranes $0.30 \mathrm{~W} / \mathrm{cm}$, corresponding to $6.60 \times 10^{-4}$ Einstein/min; ratio between volume and geometrical apparent surface of the irradiated side of the membrane $3.8 \pm 0.1 \mathrm{~cm}$; PHOTOPERM ${ }^{\circledR}$ BIT/313 membranes immobilizing $30 \pm 3$ wt. $\% \mathrm{TiO}_{2}$ ).

\begin{tabular}{|c|c|c|c|c|}
\hline Substrate & $\begin{array}{c}k_{1} \\
(\mathrm{ppm} \mathrm{C/min})\end{array}$ & $\begin{array}{c}k_{2} \\
(\mathrm{ppm} \mathrm{C/min})\end{array}$ & $\begin{array}{c}K_{1} \\
(\mathrm{ppm} \mathrm{C})^{-1}\end{array}$ & $\begin{array}{c}K_{2} \\
(\mathrm{ppm} \mathrm{C})^{-1}\end{array}$ \\
\hline Methane aqueous solution & $0.224(1)$ & $0.584(2)$ & $0.0126(3)$ & $0.0089(3)$ \\
\hline Methane gas phase & $0.222(1)$ & $0.589(4)$ & $0.013(2)$ & $0.0086(9)$ \\
\hline Ethane aqueous solution & $0.260(3)$ & $0.94(1)$ & $0.0097(3)$ & $0.0069(4)$ \\
\hline Ethane gas phase & $0.27(1)$ & $0.91(9)$ & $0.0096(6)$ & $0.009(3)$ \\
\hline$n$-heptane aqueous solution & $0.168(2)$ & $1.171(2)$ & $0.0047(2)$ & $0.0031(1)$ \\
\hline$n$-heptane gas phase & $0.174(6)$ & $1.2(2)$ & $0.0047(2)$ & $0.0031(5)$ \\
\hline
\end{tabular}

transport phenomena through the gaseous phase onto polymers, the sorption and permeation of gases and vapours in polymers have been shown to be well described over widely varying conditions by the dual-mode sorption theory [1720], in which sorption by dissolution (Henry's law) and sorption in microvoids (Langmuir isotherm) play a significant role. Factors which relate to the molecular structure of the polymer, such as polarity, hydrogen bonding, cohesive energy density, chain flexibility, steric hindrance, and cristallinity, will, among all others, have an influence on transport properties. When dealing with sorbents or reagents or catalysts immobilised onto a membrane structure, such as in the photocatalytic membranes of the present work, thermodynamics of sorption and reaction control appear to be among the most important factors in determining transport processes [21]. These reactions are ruled kinetically, and also thermodynamically, by concentration gradients, independently on diffusion and other phenomena in the bulk, either if the latter is constituted by a liquid or by a gas phase. The presence of a sorbent in a membrane structure, such as the structure which has been obtained by the photografting method used to manufacture the photocatalytic membranes employed in the present work, affords an alternative concept in membrane science and technology [13], since the endothermic step of creating a molecular size cavity in the polymer for the reacting species, either if arriving from the gas or from the liquid phase, is virtually eliminated. At the same time, the thinnest of all conceivable membranes may be realized, consisting in a monolayer where the adsorbed molecules may interact with its surroundings. Consequently, as it has been shown [13], surface diffusion and surface reactivity must be the prevailing mechanism for transport and reaction in these membranes, since a mobile adsorption layer results, able to contribute efficiently to both diffusion and reactivity [13]. This surface diffusion has been inferred by the activation energy for solubility of species, either from the gas or from the liquid phase, which nearly equals the desorption enthalpy [13]. Mass transfer, in the presence of irradiation, is accompanied by the photocatalytic reactions across these photocatalytic membranes, manufactured by the photografting nanotechnology, or by similar nanotechnologies, as those used in the present work. As a consequence, this may occur either by an activated process, most seemingly involving migration of an adsorbed unimolecular layer across the surface, or by Knudsen diffusion. The first kind of mechanism should prevail, as has been found $[14,15]$, when relatively strong adsorption interactions with active sites arise. This is in fact the case for photocatalytic membranes.

(d) The similarity of behaviour, and the coincidence of the $k$ and $K$ parameters (see Table 1 ), between experiments in the gas and in the liquid phases, is thus fully compatible with, and clearly interpreted by, the general mechanisms outlined in (c) above, as obtained and used in previous studies concerning these reactive membranes.

(e) It should be underlined, however, that consideration on rates, based exclusively on $k$ values, following a LangmuirHinshelwood model, should not be regarded as fully and absolutely reliable. In fact, it has been shown, since long time and unequivocally $[11,12]$, that also $K$ parameters have a kinetic significance, so that these latter ones are integral part of a convenient and fitting model, rather than attributing to them a truly and purely physical meaning in the sense of adsorption. This notwithstanding, a rough indication based on $k$ values can be considered, at high concentrations of substrate, as complementary to the use of the rate equation, the latter being anyway the most reliable method to treat experimental data, as has been done for example in modelling of Figures $2-7$ in which the $k K$ factor may play the most relevant role.

On a pure kinetic basis, such as that employed in the present work, it is impossible to speculate further on mechanisms. Nevertheless, this is not a limitation, because, on one 
side, mathematical modelling of kinetics is fundamental to the design of membrane photoreactors. Furthermore, on another side, the factors associated with $K$ values, both if interpreted kinetically or as a measure of adsorption-desorption equilibrium, or by both these mechanisms, play a large role [9] in determining rates and consequently overall efficiency as a function of concentration.

\section{CONCLUSIONS}

Photomineralization of model molecules of $n$-alkanes, in the gas phase and in the presence of aerosolized stoichiometric hydrogen peroxide as oxygen donor, by photocatalytic membranes immobilizing $30 \pm 3 \%$ of titanium dioxide, has been modelled by a four-parameter kinetic model [4], as successfully as has been done in a variety of cases concerning the same $n$-alkanes in aqueous solutions [9], as well as a multiplicity of other molecules in previous papers of this series. By operating in a gaseous phase saturated by water vapour, mineralisation proceeds smoothly to completeness.

The presence of a sorbent in a membrane structure, such as the structure which has been obtained by the photografting method used to manufacture the photocatalytic membranes, affords an alternative concept in membrane science and technology, since the endothermic step of creating a molecular size cavity in the polymer for the reacting species, either if arriving from the gas or from the liquid phase, is virtually eliminated. At the same time, the thinnest of all conceivable membranes may be realized, consisting in a monolayer where the adsorbed molecules may interact with its surroundings. Consequently, surface diffusion and surface reactivity must be the prevailing mechanism for transport and reaction in these membranes, since a mobile adsorption layer results, able to contribute efficiently to both diffusion and reactivity.

\section{ACKNOWLEDGMENTS}

The current paper is the 76th in a series of papers authored by Ignazio Renato Bellobono and his collaborators over the past 20 years which are collectively referred to as the "Photosynthetic Membranes" series. Some of us (RS and CC) gratefully acknowledge BIT srl (Milan, Italy), in the frame of a permanent agreement with the Polytechnic University of Bucharest, concerning research on BIT patented membranes and processes. Financial contribution by Fondazione Cariplo is also gratefully acknowledged.

\section{REFERENCES}

[1] D. F. Ollis and H. Al-Ekabi, Eds., Photocatalytic Purification and Treatment of Water and Air, Elsevier, Amsterdam, The Netherlands, 1993.

[2] J. M. Herrmann, "Water treatment by heterogeneous photocatalysis," in Environmental Catalysis, F. Jansen and R. A. van Santen, Eds., vol. 1 of Catalytic Science Series, pp. 171-194, Imperial College Press, London, UK, 1993.

[3] M. F. J. Dijkstra, E. C. B. Koerts, A. A. C. M. Beenackers, and J. A. Wesselingh, "Performance of immobilized photocatalytic reactors in continuous mode," AIChE Journal, vol. 49, no. 3, pp. 734-744, 2003.

[4] F. Ascari, I. R. Bellobono, and P. M. Tozzi, "Kinetic modelling of pilot plant photomineralization of aqueous cibacron CR as model molecule of industrial azo dyes on titanium dioxideimmobilizing membranes," Fresenius Environmental Bulletin, vol. 12, no. 10, pp. 1195-1201, 2003.

[5] I. R. Bellobono, F. Ascari, C. Lagrasta, et al., "Kinetic modelling of photomineralization of phenol, as model molecule of aromatic micropollutants, and validation of a photochemical reactor based on photocatalytic membranes immobilizing titanium dioxide and promoting photocatalysts," Fresenius Environmental Bulletin, vol. 12, no. 12, pp. 1536-1544, 2003.

[6] L. Rivas, I. R. Bellobono, and F. Ascari, "Photomineralization of $n$-alkanoic acids in aqueous solution by photocatalytic membranes. Influence of trialkyl vanadates as catalytic promoters of immobilized titanium dioxide," Chemosphere, vol. 35, no. 12, pp. 2899-2908, 1997.

[7] L. Rivas, I. R. Bellobono, and F. Ascari, "Photomineralization of $n$-alkanoic acids in aqueous solution by photocatalytic membranes. Influence of radiation power," Chemosphere, vol. 37, no. 6, pp. 1033-1044, 1998.

[8] I. R. Bellobono, C. Lagrasta, M. L. Carbonara, G. Bonizzoni, and P. M. Tozzi, "Laboratory-scale photomineralization of $n$ alkanols in aqueous solution by photocatalytic membranes immobilizing titanium dioxide," Fresenius Environmental Bulletin, vol. 12, no. 12, pp. 1545-1550, 2003.

[9] I. R. Bellobono, F. Morazzoni, R. Bianchi, et al., "Laboratoryscale photomineralisation of $n$-alkanes in aqueous solution, by photocatalytic membranes immobilising titanium dioxide," International Journal of Photoenergy, vol. 7, no. 2, pp. 79-85, 2005.

[10] I. R. Bellobono, R. Tacchi, E. Selli, and F. Muffato, "Photosynthetic membranes pure and ultrapure water production," in Membrane Separation Processes, A. Green, Ed., p. 187, BHRA, Cranfield, UK, 1989.

[11] B. Barni, A. Cavicchioli, E. Riva, et al., "Laboratory-scale photodegradation of phenol in aqueous solution by photocatalytic membranes immobilizing titanium dioxide," Chemosphere, vol. 30, no. 10, pp. 1847-1860, 1995.

[12] B. Barni, A. Cavicchioli, E. Riva, et al., "Pilot-plant-scale photodegradation of phenol in aqueous solution by photocatalytic

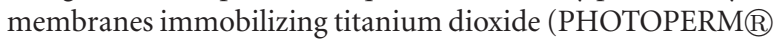
process)," Chemosphere, vol. 30, no. 10, pp. 1861-1874, 1995.

[13] I. R. Bellobono, F. Muffato, C. Ermondi, E. Selli, and L. Righetto, "Gas separation membranes containing carbon immobilized by photochemical grafting onto polymers," Journal of Membrane Science, vol. 55, no. 3, pp. 273-281, 1991.

[14] I. R. Bellobono, F. Muffato, C. Ermondi, E. Selli, L. Righetto, and M. Zeni, "Thermodynamic study of sorption of $n$-alkanes onto 5A zeolites immobilized in photosynthetic membranes," Journal of Membrane Science, vol. 55, no. 3, pp. 263-272, 1991.

[15] I. R. Bellobono, E. Selli, L. Righetto, F. Muffato, and C. Ermondi, "Thermodynamic study of adsorption of linear aliphatic monohydric alcohols and monocarboxylic acids fron n-heptane and n-decane solutions onto $\alpha$-iron(III) oxide immobilized in photosynthetic membranes," Materials Chemistry and Physics, vol. 21, no. 2, pp. 155-167, 1989.

[16] I. R. Bellobono, "Advanced oxidation and integral degradation of trichIoroethene in air by photocatalytic membranes," Life Chemistry Reports, vol. 13, p. 63, 1995.

[17] W. R. Vieth, J. M. Howell, and J. H. Hsieh, "Dual sorption theory," Journal of Membrane Science, vol. 1, no. 2, pp. 177-220, 1976. 
[18] R. T. Chern, W. J. Koros, H. B. Hopfenberg, and V. T. Stannett, "Material selection for membrane-based gas separations," Polymeric Materials Science and Engineering, vol. 50, pp. 88-92, 1984.

[19] W. J. Koros, "Simplified analysis of gas/polymer selective solubility behavior," Journal of Polymer Science: Polymer Physics Edition, vol. 23, no. 8, pp. 1611-1628, 1985.

[20] A. S. Sangani, "An application of an homogenization method to a model of diffusion in glassy polymers," Journal of Polymer Science, Part B: Polymer Physics, vol. 24, no. 3, pp. 563-575, 1986.

[21] I. R. Bellobono, F. Muffato, E. Selli, L. Righetto, and R. Tacchi, "Transport of oxygen facilitated by peroxo-bis $\left[N, N^{\prime}\right.$ ethylene bis-(salicylideneiminato)-dimethylformamide-cobalt (III)] embedded in liquid membranes immobilized by photografting onto cellulose," Gas Separation \& Purification, vol. 1, no. 2, pp. 103-106, 1987. 


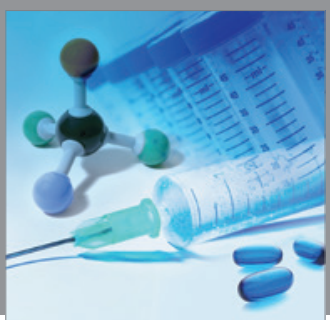

International Journal of

Medicinal Chemistry

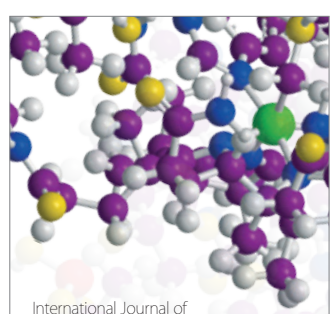

Carbohydrate Chemistry

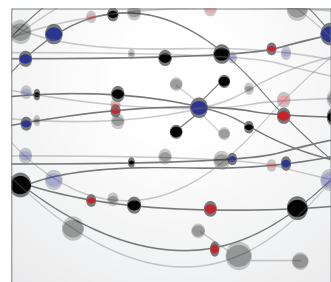

The Scientific World Journal
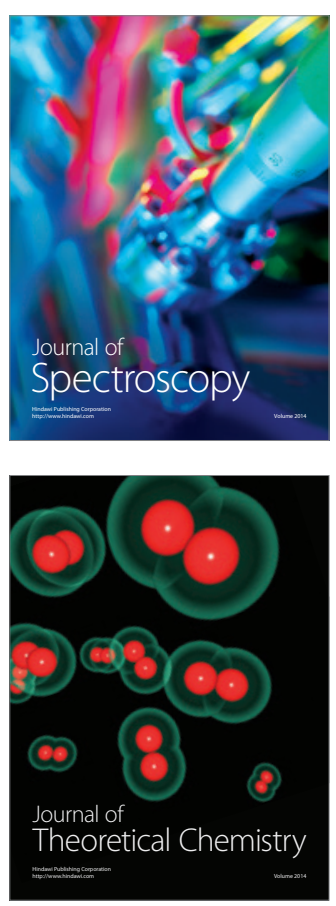
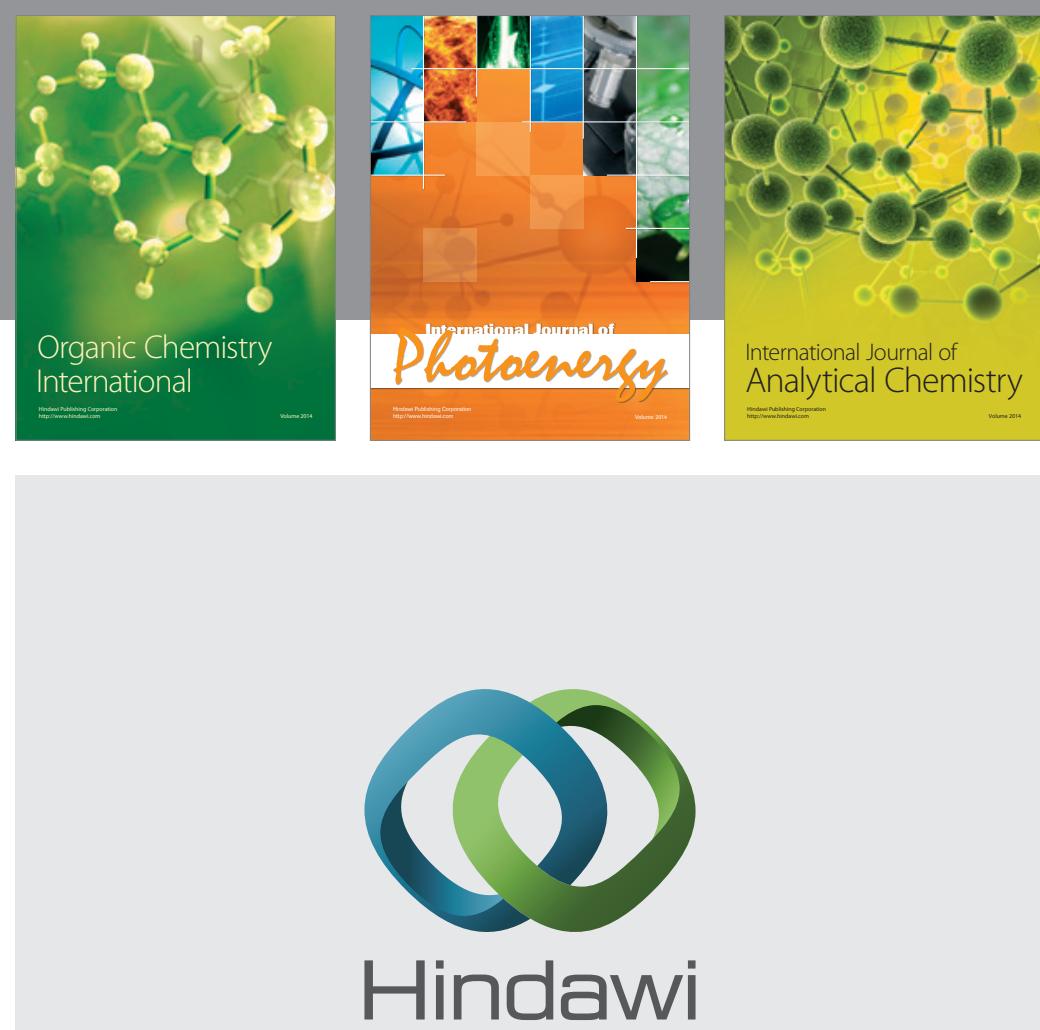

Submit your manuscripts at

http://www.hindawi.com
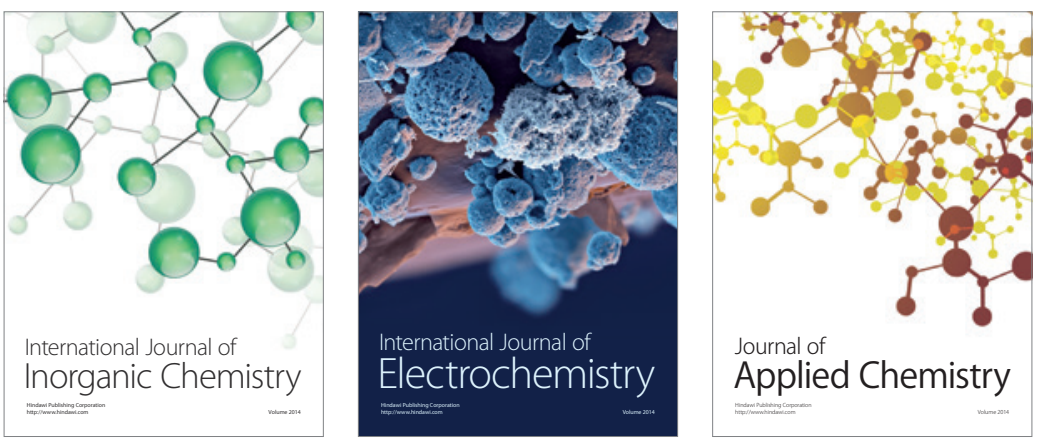

Journal of

Applied Chemistry
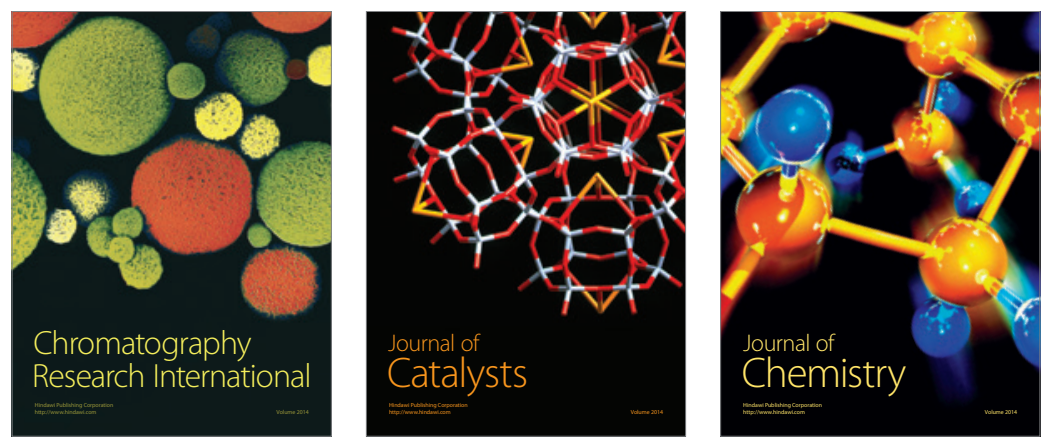
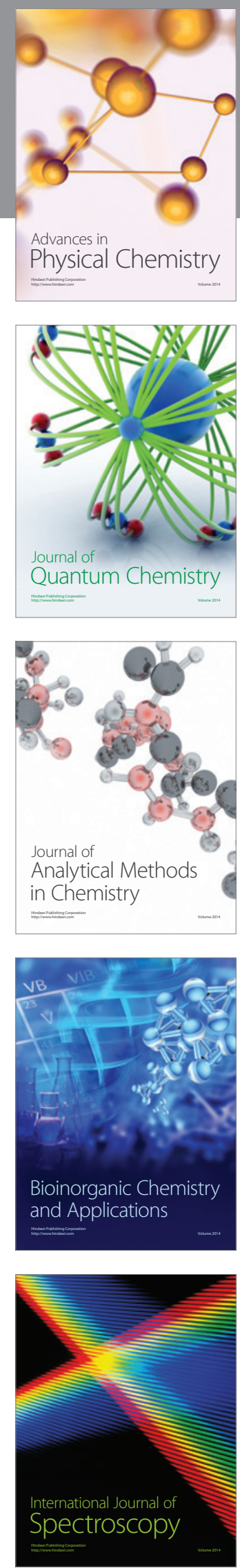